\title{
Analysis and simulation of standing wave pattern of powerful HF radio waves in ionospheric reflection region
}

\author{
Chen Wang, Chen Zhou*, Zheng-Yu Zhao and Xu-Bo Yang
}

\begin{abstract}
For the study of the various non-linear effects generated in ionospheric modulation experiments, accurate calculation of the field intensity variation in the whole reflection region for an electromagnetic wave vertically impinging upon the ionosphere is meaningful. In this paper, mathematical expressions of the electric field components of the characteristic heating waves are derived, by coupling the equation describing a wave initially impinging vertically upon the ionosphere with the Forsterling equation. The variation of each component of the electric field and the total electric field intensity of the standing wave pattern under a specific density profile are calculated by means of a uniform approximation, which is applied throughout the region near the reflection point. The numerical calculation results demonstrate that the total electric field intensity of the ordinary (O)-mode wave varies rapidly in space and reaches several maxima below the reflection point. Evident swelling phenomena of the electric field intensity are found. Our results also indicate that this effect is more pronounced at higher latitudes and that the geomagnetic field is important for wave pattern variation. The electric field intensity of the standing wave pattern of the extraordinary $(X)$-mode wave exhibits some growth below the reflection point, but its swelling effect is significantly weaker than that of the O-mode wave.
\end{abstract}

Keywords: Forsterling function; Uniform approximation; Field intensity of standing wave pattern

\section{Background}

For many years, active remote probing by means of high-frequency (HF) radio waves has been a standard technique for diagnosing the ionosphere. This is because the recording and analysis of the reflected or scattered part of the HF radiation constitutes a convenient method of determining a number of ionospheric parameters or of investigating various physical processes occurring in the ionospheric plasma. The ionosphere is also treated as a natural space plasma laboratory and modulated more actively using high-power HF pump waves, so as to study the interactions of the electromagnetic waves and plasma. Research attempts in this area began with the Platteville heating experiments conducted in Colorado, USA, in the 1960s (Utlaut, 1970; Utlaut and Cohen, 1971; Utlaut and Violette, 1972). In ionospheric modification experiments, a powerful $\mathrm{HF}$ electromagnetic wave incident on the ionosphere can

\footnotetext{
* Correspondence: chen.zhou.whu@gmail.com

Department of Space Physics, School of Electronic Information, Wuhan University, Wuhan 430079, China
}

produce nonlinear effects on time scales ranging from tens of microseconds to minutes, and on size scales ranging from meters to kilometers. Strong nonlinear processes including self-focusing, parametric and resonant instability, and accompanying phenomena such as enhanced airglow production, Langmuir turbulence (LT), and the generation of geomagnetic-field-aligned density irregularities (FAI) have been found to occur (Gondarenko et al. 2003; Stubbe et al. 1984).

A characteristic feature of the instabilities excited during ionospheric modulation is the existence of a finite threshold value of the HF pump electric field $\vec{E}$ intensity, which must be exceeded for these instabilities to be excited (Fejer, 1979; Fejer, 1981; Fejer et al. 1983). In order to correctly interpret the observations made in these experiments, it is therefore essential to be able to accurately calculate the $\vec{E}$ wave pattern in the whole reflection region.

In this regard, the spatial distributions of the pumpwave $\vec{E}$ are derived using a wave equation, which is in

\section{Springer}


turn derived within the WKB or geometrical optics approximation. However, it is impossible to obtain the $\vec{E}$ value in the vicinity of the reflection (conversion) point, where the WKB approximation breaks down (Ginzburg, 1970). A purely numerical simulation method for obtaining the variation of the pump-wave $\vec{E}$ in the whole reflection region is used by Gondarenko et al. 2004. In addition, the ray tracing method proposed by Field et al. 1990 and Hinkel et al. 1993 yields the spatial distribution of the pump-wave $\vec{E}$ by accurately calculating the phase of the pump-wave propagation path. An ingenious analytical method devised by Lundborg and Thide 1985, 1986 is used to calculate the variation of the characteristicwave $\vec{E}$ near the reflection region, but only ordinary (O)-mode waves are considered. As regards Chinese scholars, the empirical model is mostly used directly, to estimate the spatial distributions of the pump-wave $\vec{E}$; however, these estimates are accurate to within an order of magnitude only (Huang and Gu, 2003; Hao et al. 2013). Of course, purely numerical methods can obtain the variation of the pump-wave $\vec{E}$; however, the numerical models are very complex and the computational requirements are high. In addition, analytical methods always provide more information about the solution than pure numbers, and analytic formulas are very quickly evaluated, even on a moderately sized desktop computer.

We have therefore adopted the "uniform approximation" analytical method, which is similar to the method used by Lundborg and Thide 1986, to derive accurate approximations for the variation of the $\vec{E}$ of both the $\mathrm{O}$ and the extraordinary $(\mathrm{X})$-mode characteristic waves. In contrast to the similar WKB or phase integral approximations, these approximations do not break down in the reflection region (Langer, 1937; Miller and Good, 1953). In section 2, we provide the mathematical expressions of the $\vec{E}$ components of both characteristic mode waves, which are derived using an approach that couples the equation describing a wave initially impinging vertically upon the ionosphere with the Forsterling equation. Then, analytic solutions of each component calculated using the "uniform approximation" method are presented in section 3 . In section 4 , we present the numerical results for the $\vec{E}$ intensity variation of the standing wave pattern of the $\mathrm{O}$ - and X-mode characteristic waves, under a specific density profile and throughout the whole reflection region (including the upper-hybrid resonance altitude). These calculations are conducted for different latitudes and at different local times in one location. Along with the real parts of the effective refractive index functions for the $\mathrm{O}$ - and $\mathrm{X}$-mode waves, the swelling factors for both characteristic mode waves are also calculated. The field strength obtained for an unmagnetized plasma is given last and compared with the previously obtained results. Finally, our conclusions are outlined in section 5 .

\section{Wave formulation}

The general equation for wave propagation in an isotropic inhomogeneous plasma medium can be derived from the Maxwell equations, which are expressed in the Cartesian coordinate system in the form (Gurevich, 1978):

$$
\Delta \vec{E}-\nabla(\nabla \bullet \vec{E})+\frac{\omega^{2}}{c^{2}} \varepsilon^{\prime}(\omega, r) \vec{E}=0
$$

where $\varepsilon^{\prime}(\omega, r)=\varepsilon(\omega)-i \frac{4 \pi}{\omega} \sigma(\omega), \sigma$ is the conductivity tensor and $\omega$ is the wave angular frequency. When considering a plane electromagnetic wave impinging vertically upon the ionosphere, the changes in $\vec{E}$ depend on the $z$-coordinate only. Then, Eq. (1) can be rewritten as:

$$
\frac{d^{2} E}{d z^{2}}+\frac{\omega^{2}}{c^{2}} \varepsilon^{\prime}(\omega, z) E=0
$$

This equation is applicable to both of the horizontal $\vec{E}$ components, $E_{x}$ and $E_{y}$. When the background ionosphere is considered to be a linear plasma layer without absorption, Eq. (2) becomes:

$$
\varepsilon^{\prime}(\omega, z)=\varepsilon(z)=n^{2}(z), \sigma=0 .
$$

where $n^{2}$ is the square of the complex refractive index. $E_{x}$ and $E_{y}$ are determined from the one-dimensional time-independent wave equation:

$$
\frac{d^{2} E}{d z^{2}}+k^{2} n^{2}(z) E=0
$$

where $k=\omega / c$.

In this paper, we are interested in analyzing the variation of the $\vec{E}$ intensity near the reflection point caused by the initial vertical propagation of the HF radio waves in the ionosphere. We therefore model the ionosphere as a cold, magnetoactive, collisional plasma. In the right-handed Cartesian coordinate system we have chosen, the $x$-, $y$-, and $z$-axes respectively point toward magnetic east, magnetic north, and vertically upward, that is, the $z$-axis parallel to the $k$ vector of the radio wave launched from a transmitter on the ground. The geomagnetic field $B_{0}$ is considered to lie in the yoz-plane and to create an angle $\theta$ to the negative $z$-axis, as shown in Fig. 1. We assume that the standard magnetoionic notations are suitable for our model. Then, we have (Rishbeth and Garriott, 1969): 


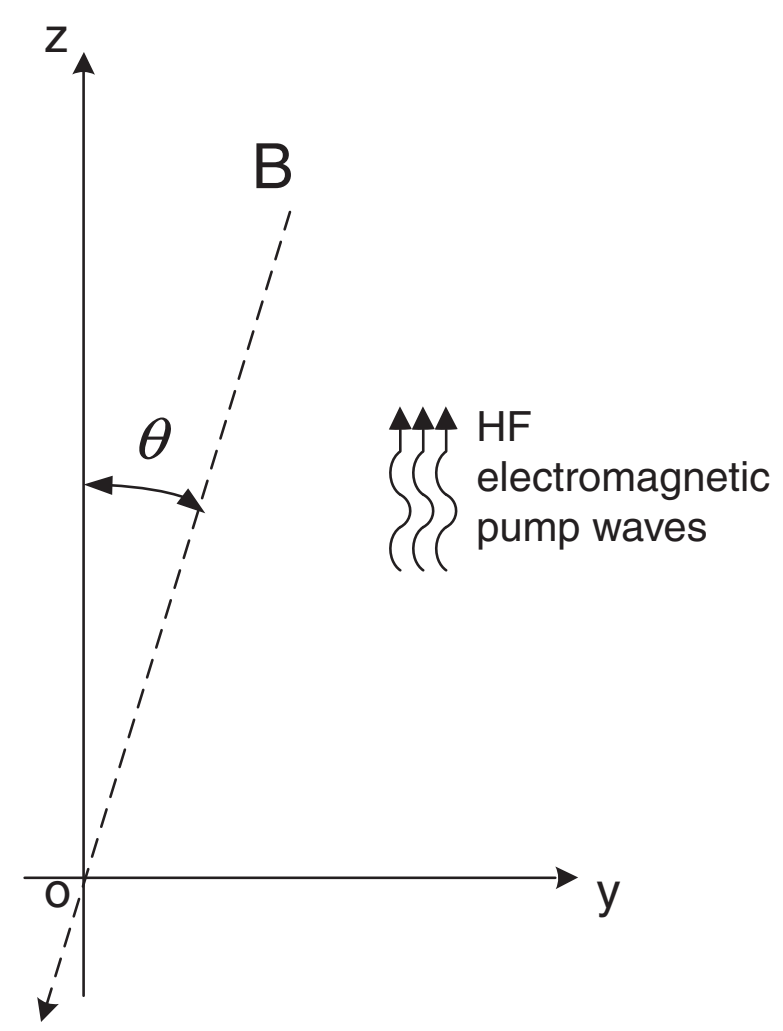

Fig. 1 Schematic diagram of Cartesian coordinate system used in this paper

$$
X=\omega_{p e}^{2} / \omega^{2}, Y=\omega_{c e} / \omega, Z=\nu / \omega
$$

where $\omega_{p e}$ and $\omega_{c e}$ are the electron plasma (angular) frequency and the electron cyclotron frequency, respectively. For the specific expressions of these two terms, refer to Rishbeth and Garriott 1969. The electron collision frequency $v_{e}=v_{e m}+v_{e i}$, where $v_{e m}$ and $v_{e i}$ are the collision frequencies of electrons with neutral particles and with ions, respectively. The specific expressions of these collision frequencies are given in detail in Banks and Kocharts 1973 and Schunk and Walker 1980.

The wave equations for the components of $\vec{E}$ can be derived from Maxwell's equations in the usual, wellknown way, as we have discussed above. Assuming a time variation of $\exp [-i \omega t]$, we obtain the following wave equations (Ginzburg, 1970):

$$
\begin{aligned}
& \frac{d^{2} E_{x}}{d z^{2}}+k^{2} Q_{11}(z) E_{x}+k^{2} Q_{12}(z) E_{y}=0, \\
& \frac{d^{2} E_{y}}{d z^{2}}+k^{2} Q_{21}(z) E_{x}+k^{2} Q_{22}(z) E_{y}=0,
\end{aligned}
$$

$$
E_{z}+Q_{31}(z) E_{x}+Q_{32}(z) E_{y}=0,
$$

where the functions $Q_{i j}(z)$ are given by

$$
\begin{aligned}
Q_{11}(z)= & 1-\frac{X(z)[1+i Z][1+i Z-X(z)]}{D(z)} \\
Q_{12}(z)= & -Q_{21}(z)=-i \frac{X(z)[1+i Z-X(z)] Y \cos \theta}{D(z)} \\
Q_{22}(z)= & 1-\frac{X(z)[1+i Z][1+i Z-X(z)]}{D(z)} \\
& +\frac{X(z) Y^{2} \sin ^{2} \theta}{D(z)} \\
Q_{31}(z)= & i \frac{X(z)[1+i Z] Y \sin \theta}{D(z)} \\
Q_{32}(z)= & -\frac{X(z) Y^{2} \sin \theta \cos \theta}{D(z)}
\end{aligned}
$$

with the common denominator

$$
D(z)=[1+i Z-X(z)]\left[(1+i Z)^{2}-Y^{2}\right]-X(z) Y^{2} \sin ^{2} \theta .
$$

We can easily see that wave Eqs. (6a) and (6b) are coupled and, therefore, it is a formidable task to obtain exact solutions in their current form. However, for a homogeneous medium, the exact solution of (6a) and (6b) can be obtained trivially by solving the eigenvalue problem of the corresponding matrix, which is then constant. This yields the eigenvalues (Lundborg and Thide, 1986):

$$
\begin{aligned}
n_{O / X}^{2}=1 & -\frac{X}{2 D}\left\{2(1+i Z)(1+i Z-X)-Y^{2} \sin ^{2} \theta\right. \\
& \left.\mp\left[Y^{4} \sin ^{4} \theta+4(1+i Z-X)^{2} Y^{2} \cos ^{2} \theta\right]^{\frac{1}{2}}\right\},
\end{aligned}
$$

and the corresponding eigenvectors described by the transverse polarization

$$
\begin{aligned}
\rho=E_{y} / E_{x} & \\
\rho_{O / X}= & \frac{i}{2(1+i Z-X) Y \cos \theta}\left\{Y^{2} \sin ^{2} \theta\right. \\
& \left. \pm\left[Y^{4} \sin ^{4} \theta+4(1+i Z-X)^{2} Y^{2} \cos ^{2} \theta\right]^{\frac{1}{2}}\right\} .
\end{aligned}
$$

The lower subscripts and signs $\mp$ (or \pm ) in Eqs. (8) and (9) correspond to the $\mathrm{O}$ and $\mathrm{X}$ modes, respectively. From Eq. (9), we easily find that the two polarizations satisfy $\rho_{O} \rho_{X}=1$. For convenience in what follows, we primarily use the quantity $\rho_{X}$ in our equations, as $\rho_{O}$ becomes very large in the $\mathrm{O}$-mode reflection region. 
The characteristic waves in the homogeneous medium are thus the well-known $\mathrm{O}$ - and $\mathrm{X}$-mode wave with complex wave numbers $k n_{O}$ or $k n_{X}$, according to (8), and with polarizations given by (9). However, it must be remembered that, in an inhomogeneous medium, these waves are no longer exact solutions of the wave equations. Obviously, the pump-wave reflection area in our calculation no longer satisfies this condition. However, if the medium is slowly varying, one might hope that there exist approximate solutions under certain conditions corresponding to the characteristic modes that are at least less strongly coupled to each other than the Cartesian field components. It might therefore be a good idea to transform the dependent variables $E_{x}$ and $E_{y}$ in (6a) and (6b) to new variables corresponding to the characteristic wave modes for a homogeneous plasma. The specific conversion procedure is described in both Lundborg and Thide 1986 and Budden 1966. Hence, we express the transverse field in terms of the two Forsterling functions $F_{O}$ and $F_{X}$, where:

$$
\begin{aligned}
& E_{x}=\rho_{X} E_{y, O}+E_{x, X}, E_{y}=E_{y, O}+\rho_{X} E_{x, X}, \\
& E_{y, O}=\left(\rho_{X}^{2}-1\right)^{-\frac{1}{2}} F_{O}, E_{x, X}=\left(\rho_{X}^{2}-1\right)^{-\frac{1}{2}} F_{X} .
\end{aligned}
$$

The new variables $F_{O}$ and $F_{X}$ in Eq. (10) must satisfy

$$
\begin{aligned}
& F_{O}^{\prime \prime}+\left(k^{2} n_{O}^{2}+q^{2}\right) F_{O}=q^{\prime} F_{X}+2 q F_{X}^{\prime}, \\
& F_{X}^{\prime \prime}+\left(k^{2} n_{X}^{2}+q^{2}\right) F_{X}=q^{\prime} F_{O}+2 q F_{O}^{\prime},
\end{aligned}
$$

where the coupling function $q$ is defined as

$$
q=\frac{i\left(i Z^{\prime}-X^{\prime}\right) Y \cos \theta \sin ^{2} \theta}{Y^{2} \sin ^{4} \theta+4(1+i Z-X)^{2} \cos ^{2} \theta}
$$

Equations (11a) and (11b) are the Forsterling equations, which contain no approximations and, hence, are equivalent to the original equations, (6a) and (6b). Assuming the solutions of Eqs. (11a) and (11b) are known, we obtain $E_{x}$ and $E_{y}$ from Eq. (10) and, finally, $E_{z}$ from Eq. (6c). We can then achieve a formal simplification of these results by introducing the longitudinal polarization

$$
\rho_{L}=E_{z} / E_{x}
$$

Substituting (13) into Eqs. (6c) and (10) yields

$$
\rho_{L, O / X}=\frac{i Y \sin \theta}{1+i Z-X}\left(n_{O / X^{2}}^{2}\right) .
$$

We may now write the exact total field in the form

$$
E=E_{O}+E_{X}
$$

where

$$
\begin{aligned}
E_{O} & =\left(1, \rho_{O}, \rho_{L, O}\right) \rho_{X}\left(\rho_{X}^{2}-1\right)^{-\frac{1}{2}} F_{O} \\
& =\left(\rho_{X}, 1, \rho_{X} \rho_{L, O}\right)\left(\rho_{X}^{2}-1\right)^{-\frac{1}{2}} F_{O} \\
E_{X} & =\left(1, \rho_{X}, \rho_{L, X}\right)\left(\rho_{X}^{2}-1\right)^{-\frac{1}{2}} F_{X} .
\end{aligned}
$$

\section{Methods}

The new approach presented here describes the $\vec{E}$ components of the characteristic waves by utilizing the Forsterling functions discussed above. For our purposes, the wave Eqs. (11a) and (11b) are in a more suitable form than Eqs. (6a) and (6b), since the coupling, which is expressed in terms of $q$, is sufficiently small in our applications to be neglected. Indeed, according to Eq. (12), $q$ is proportional to $i Z^{\prime}-X^{\prime}$, which is very small in the slowly varying medium we have chosen. Hence, in such a medium, Eqs. (11a) and (11b) can in good approximation be treated as two uncoupled equations, where

$$
\begin{aligned}
& F_{O}^{\prime \prime}+k^{2} n_{O}^{2} F_{O}=0, \\
& F_{X}^{\prime \prime}+k^{2} n_{X}^{2} F_{X}=0 .
\end{aligned}
$$

These are the wave equations for the well-known characteristic modes, i.e., the $\mathrm{O}$ and $\mathrm{X}$ modes, respectively. It can be seen that the forms of these wave equations are almost exactly identical to that of Eq. (4). In the present paper, these equations are treated using a more comprehensive and versatile uniform approximation method, which can provide accurate solutions that are valid throughout entire reflection regions.

In order to apply this method, we consider a differential equation in the following form (Berry and Mount, 1972):

$$
\frac{d^{2} \psi}{d \phi^{2}}+k^{2} P^{2}(\phi) \psi=0
$$

where $\phi(z)$ is the mapping function, $P$ is the phase value of the $\phi(z)$ and $\psi$ is the potential value, the solutions of $\psi$ has known and Eq. (17) is our so-called "comparison equation". Next, we express the solutions of wave Eq. (16a) in terms of the known $\psi$ in the form:

$$
F_{O}(z)=\left(\frac{d \phi(z)}{d z}\right)^{-\frac{1}{2}} \psi(\phi(z))
$$

and the mapping function $\phi(z)$ satisfies the equation 


$$
n^{2}(z)=\left(\frac{d \phi}{d z}\right)^{2} P^{2}(\phi)-\frac{1}{k^{2}}\left(\frac{d \phi}{d z}\right)^{\frac{1}{2}} \frac{d^{2}}{d z^{2}}\left(\frac{d \phi}{d z}\right)^{-\frac{1}{2}}
$$

exactly. Thus, Eq. (18) gives the exact solution of $F_{O}(z)$. If $P^{2}(\phi)$ is appropriately chosen, $\phi(z)$ will be slowly varying and the second term on the right-hand side of $n^{2}(z)$ may be neglected on account of the first. Hence, we obtain the first approximation

$$
\frac{d \phi}{d z}=\frac{n(z)}{P(\phi)}
$$

As this method functions only if the transition points of the two wave equations correspond to each other, we may define $\phi(z)$ implicitly, using Eq. (17), from

$$
\zeta=k \int_{\phi_{1}}^{\phi} P\left(\phi^{\prime}\right) d \phi^{\prime}=k \int_{t_{1}}^{z} n\left(z^{\prime}\right) d z^{\prime} .
$$

Here, we introduce the intermediary variable $\zeta=\zeta(\phi)$ $=\zeta(\phi(z))$. Hence, $\phi_{1}$ is obviously the zero (transition point) of $P^{2}(\phi)$ that corresponds to the zero $t_{1}$ of $n^{2}(z)$.

In the case of only a single complex reflection point, we can choose the Airy equation as our comparison equation, where:

$$
\frac{d^{2} \psi}{d \phi^{2}}-\phi \psi=0
$$

Here, we have $k^{2} P^{2}(\phi)=-\phi$, with the zero $\phi_{1}=0$ corresponding to the zero $t_{1}$ of $n^{2}(z)$ in Eq. (4). By means of Eqs. (17) and (21), we then obtain

$$
\phi(z)=\left[-\frac{3 i}{2} \zeta\right]^{\frac{2}{3}}
$$

The general solution of Eq. (17) can be expressed in terms of the Airy functions (Abramowitz and Stegun, 1972), such that:

$$
\psi(\phi)=\alpha A i(-\operatorname{Re} \phi(z))+\beta B i(-\operatorname{Re} \phi(z)) s
$$

where $\alpha$ and $\beta$ are the normalizing factor and the Stokes coefficient respectively, and the factor $\alpha$ is defined as (Hinkel et al. 1992):

$$
\alpha=2\left[k \pi\left(\rho_{X}^{2}\left(z_{n}\right)-1\right)\right]^{1 / 2} E_{n} \exp \left\{i\left[k \int_{z_{n}}^{z_{0}} n_{O}(z) d z-\frac{\pi}{4}\right]\right\} .
$$

Here, $z_{n}$ is the base point of the ionosphere and the value of $E_{n}$ is the field intensity at $z_{n}$, which is determined by the effective radiated power (ERP) value and the non-deviation absorption of the HF pump waves. The physical conditions require the solution $F_{O}(z)$ of Eq. (18) to decay as $z \rightarrow \infty$ and, hence, we must choose a $\psi$ solution that decays in the $|\arg \phi|<\frac{\pi}{3}$ region, i.e., we require $\beta=0$. From Eqs. (18), (19), and (24), we thus obtain the first-order uniform solution of $F_{O}(z)$, such that:

$$
F_{O}(z)=\alpha\left[\frac{-\phi(z)}{k^{2} n_{O}^{2}(z)}\right] A i(-\operatorname{Re} \phi(z)) .
$$

As we have already mentioned, the solution technique utilizing a uniform approximation of the wave equation, what we discussed above can also be used to treat the $\mathrm{X}$ mode.

\section{Results and discussion}

The approximation method discussed above can only be employed to derive the solutions for the particular case having one complex zero of $n^{2}(z)$. This is because the applicability of the uniform approximation method is inherently connected to the behaviors of functions (19) and (21). The O-mode refractive index has a zero when

$$
X=1+i Z,
$$

i.e., at the same points as for a corresponding nonmagnetic medium. The X-mode refractive index is zero when

$$
X=1+i Z-Y .
$$

In this paper, we only analyze the variation of the field intensities of the HF pump-wave standing wave patterns for over-dense heating mode. In this case, the emission frequencies of powerful pump waves from the transmitter are less than the critical frequency value of the ionospheric F2 layer $f_{0} F_{2}$, and the pump-wave reflection heights are lower than the density peak of the F2 layer $h_{m} F_{2}$. As the pump-wave reflection heights in our calculation lie in the $\mathrm{F}$ layer of the ionosphere, where the electron density monotonically increases with height, we assume that the electron density profile is a linear monotonic case, such that:

$$
X=1+\frac{z-z_{0}}{h},
$$

where $z_{0}$ is the reflection point (the real part of the complex transition point) of $\mathrm{O}$-mode waves and $h$ is the scale length of the profile.

From the $E_{y}$ and $E_{z}$ components of $\vec{E}$ in Eq. (15), we obtain its components perpendicular and parallel to the geomagnetic field as

$$
\begin{aligned}
& E_{\perp}=\cos \theta E_{y}+\sin \theta E_{z}, \\
& E_{\|}=-\sin \theta E_{y}+\cos \theta E_{z},
\end{aligned}
$$

respectively. We now calculate the variation of the field components in Eqs. (15), (29), and (30), for the linear electron density profile we assumed in Eq. (28). The 
parameters chosen for our simulation are listed in detail for three locations in Table 1.

As shown above, not only the parameters of the $\mathrm{F}$ region at Alaska, Wuhan, and Haikou (three daytime $(\mathrm{LT}=1400)$ locations corresponding to different latitude areas) were chosen for our calculation but also the parameters of the Wuhan area at night $(\mathrm{LT}=0200)$. The latter were selected in order to study the impact of the day-night differences in the ionospheric background parameters on the distribution of the HF pump waves. Here, the emission frequency of the pump wave was chosen to be 0.9 times the local $f_{0} F_{2}$, and the ERP of the pump waves was set to $10 \mathrm{MW}$. Note that the upperhybrid resonance altitude listed in the last line of Table 1 was included in the calculation interval for the O-mode waves, as many nonlinear phenomena, such as fieldaligned irregularities, optical emissions, and stimulated electromagnetic emissions, are influenced by the upperhybrid resonance. This resonance occurs at the altitude where the upper-hybrid resonance frequency $\omega_{U H}$ equals $\omega$, and $\omega_{U H}^{2}=\omega_{p e}^{2}+\omega_{c e}^{2}$. The simulation results for the three locations in daytime are shown in Figs. 2, 3, and 4, while the real parts of the refractive index function for the $\mathrm{O}$ and $\mathrm{X}$ modes $n_{O / X}^{2}(z)$ are also presented in the Figs. 2a, c, 3a, c, and 4a, c.

The red dashed vertical line in Fig. 2a represents the location of the upper-hybrid resonance altitude.

In Fig. 2a, the real part of $n_{O}^{2}$ is far from linear. The function is rather smooth, whereas its derivative undergoes a rapid change in the region around $z_{0}$, where the

Table 1 Selected parameters for linear profile model of $F$ region at Alaska, Wuhan, and Haikou

\begin{tabular}{|c|c|c|c|c|}
\hline \multirow[t]{2}{*}{ Time } & \multicolumn{4}{|c|}{ March 15, 2006} \\
\hline & LT1400 & LT1400 & LT0200 & LT1400 \\
\hline Location & Alaska & Wuhan & & Haikou \\
\hline $\begin{array}{l}\text { Latitude and } \\
\text { longitude }\end{array}$ & $\begin{array}{l}62.39^{\circ} \mathrm{N} \\
145.15^{\circ} \mathrm{W}\end{array}$ & $\begin{array}{l}30.5^{\circ} \mathrm{N} \\
114.35^{\circ} \mathrm{E}\end{array}$ & & $\begin{array}{l}20.01^{\circ} \mathrm{N}, \\
110.35^{\circ} \mathrm{E}\end{array}$ \\
\hline$\theta$, deg & 14.19 & 44.47 & 44.47 & 62.83 \\
\hline$f_{0} F_{2}, \mathrm{MHz}$ & 4.9933 & 8.2806 & 5.6387 & 9.5937 \\
\hline$\omega /(2 \pi), \mathrm{MHz}$ & 4.494 & 7.4526 & 5.0748 & 8.6343 \\
\hline$\omega_{c e} /(2 \pi), \mathrm{MHz}$ & 1.4169 & 1.2035 & 1.1996 & 1.0869 \\
\hline$v /(2 \pi), \mathrm{Hz}$ & 309.7 & 695.8 & 316.9 & 955.4 \\
\hline$h, \mathrm{~km}$ & 78.2 & 96.3 & 57.3 & 118.8 \\
\hline$z_{0}, \mathrm{~km}$ & 209 & 221 & 267 & 237 \\
\hline$Z_{U H}, \mathrm{~km}$ & 204.02 & 218.49 & 263.29 & 235.12 \\
\hline
\end{tabular}

From top to bottom, the given parameters are the latitude and longitude of the selected locations, the angle between the geomagnetic field and the downward vertical, the critical frequency of the ionospheric F2 layer, the wave frequency, electron cyclotron frequency in the geomagnetic field, the effective collision frequency, the scale height, the reflection height of O-mode wave, and the upper-hybrid resonance altitude value of $n_{O}^{2}$ has a zero $\left(z=z_{0}=209 \mathrm{~km}\right.$; the black circle shown in the figure). However, the derivative remains monotonic. Furthermore, we find that the refractive index of the X-mode waves $n_{X}^{2}$ goes to infinity before the O-mode wave reflection point $(z=208.4 \mathrm{~km}$; the black dashed vertical line in Fig. 2a); this is referred to as the plasma resonance region. It can be seen that the $\vec{E}$ components of the O-mode waves vary rapidly in the region before $z_{0}$. The $\vec{E}$ component parallel to the geomagnetic field $E_{\|}$plays a dominant role in all components and undergoes obvious growth (the blue solid curve in Fig. 2b), which leads to a rapid increase in the total $\vec{E}$ intensity; this is usually referred to as swelling. At the same time, the component perpendicular to the geomagnetic field $E_{\perp}$ decreases rapidly in the region near $z_{0}$ (the black solid curve in Fig. 2b), and almost vanishes at $z_{0}$ itself. It is also found that the total electric field $|E|$ (the red solid curve in Fig. 2 b) varies more rapidly and has a larger amplitude than the electric field for the isotropic case $\left|E_{0}\right|$ (the green dashed curve in Fig. 2b), when the geomagnetic field is neglected. Here, we define the pump-wave swelling factor, which is the ratio of the maximum $\vec{E}$ amplitudes before the reflection height to the empirical value. The latter is normally calculated using the empirical model, as a function of altitude from the pump-wave ERP, i.e., $E \approx 0.25 \sqrt{E R P} / z$ (Gurevich, 1978; Robinson, 1989). The swelling factor of the O-mode waves for the Alaska case is approximately 10.97 .

In Fig. 2c, the value of $n_{X}^{2}$ has a zero before the reflection point of the $\mathrm{O}$-mode waves $(z=184.3 \mathrm{~km}$, the black circle shown in the figure), indicating that the X-mode waves do not usually reach the reflection level of the Omode waves. The $\vec{E}$ components of the X-mode waves exhibit significantly different behavior, in that $E_{\|}$(the blue solid curve in Fig. 2d) is significantly smaller than the other two components, $E_{\perp}$ and $E_{x}$ (indicated by the black and green solid curves in Fig. 2d, respectively), which are perpendicular to the geomagnetic field. All $\vec{E}$ components of the $\mathrm{X}$-mode waves are not enhanced significantly in the vicinity of its reflection point, which results in a reduced field swelling effect. The swelling factor for the X-mode waves in Alaska is approximately 4.05 .

From Eq. (9), we can see that the value of $\rho_{O}$ is approximately equal to $i$ for variable $z$ is not too close to the $z_{0}$ and $Y \ll 1$. Hence, the transverse part of the upgoing O-mode waves is, to a good approximation, circularly polarized in the right-hand sense. On the other hand, for $z$ value that is very close to the $z_{0}, X \approx 1, n_{O}^{2} \approx 0$, and $Z \ll Y$, and we can obtain the following approximation from Eqs. (9) and (14) 


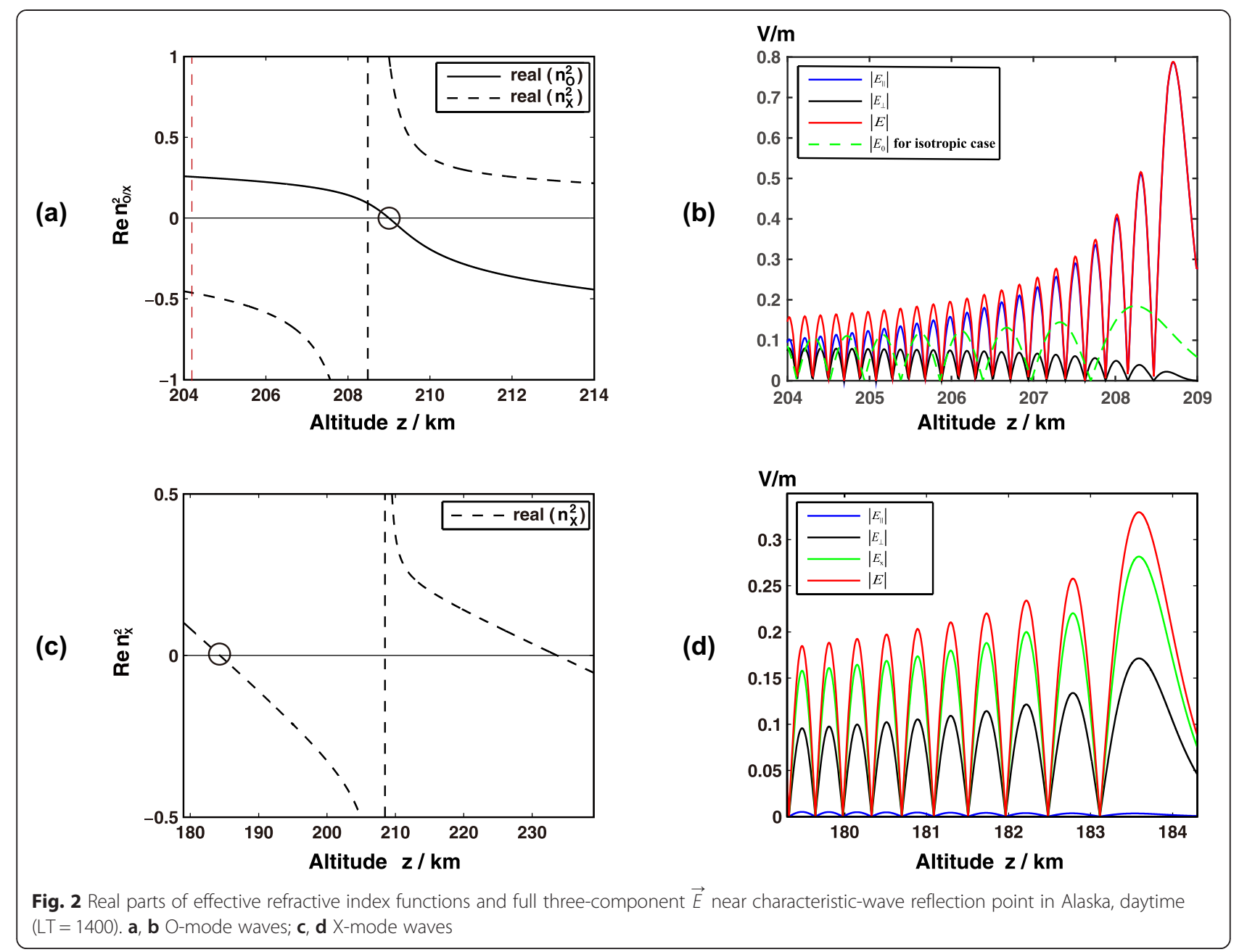

$$
\rho_{X} \approx \frac{Z \cos \theta}{Y \sin ^{2} \theta}, \rho_{L, O} \approx-\frac{Y \sin \theta}{Z} .
$$

According to Eqs. (15), (29), and (30), this means that at exactly $z_{0}$ and in its immediate vicinity

$$
\begin{aligned}
E_{\|} & =-\sin \theta E_{y}+\cos \theta E_{z} \\
& =-\sin \theta E_{y}+\cos \theta \cdot \rho_{X} \rho_{L O} E_{y} \\
& \approx\left(-\sin \theta-\frac{\cos ^{2} \theta}{\sin \theta}\right) E_{y}=-\frac{1}{\sin \theta} E_{y}
\end{aligned}
$$

Analogously,

$$
\begin{aligned}
E_{\perp} & =\cos \theta E_{y}+\sin \theta E_{z} \\
& =\left(\cos \theta+\sin \theta \bullet \rho_{X} \rho_{L O}\right) E_{y} \\
& \approx\left(\cos \theta-\sin \theta \frac{\cos \theta}{\sin \theta}\right) E_{y}=0 .
\end{aligned}
$$

The above discussion gives a distinct explanation of why the $E_{\perp}$ component goes to zero at $z_{0}$, and shows that $\vec{E}$ becomes almost aligned with the geomagnetic field very near $z_{0}$. In addition, its propagation vector becomes perpendicular to the geomagnetic field $B_{0}$, also because of this field orientation near $z_{0}$. Thus, the electromagnetic wave can easily couple to electrostatic mode waves such as Langmuir or ion-acoustic waves, resulting in various instabilities (Ginzburg, 1970; Rietveld et al. 1993).

For X-mode waves, also from Eq. (28), we can see that for $z$ values very close to the $X$-mode wave reflection point $z_{0}-h Y$, then $X \rightarrow 1-Y$, and $|1-X| \simeq Y$. Therefore, we can obtain the approximation from Eqs. (9) and (14), such that:

$$
\rho_{X} \approx-i \cos \theta, \rho_{L, X} \approx-\frac{i Y \sin \theta}{Y+i Z} .
$$

The equations above clearly indicate that $\left|\rho_{X}\right|<1$ and $\left|\rho_{L, X}\right|<1$. Therefore, combining these expressions with Eq. (15) may give a distinct explanation of why the $\vec{E}$ component perpendicular to the magnetic meridian 


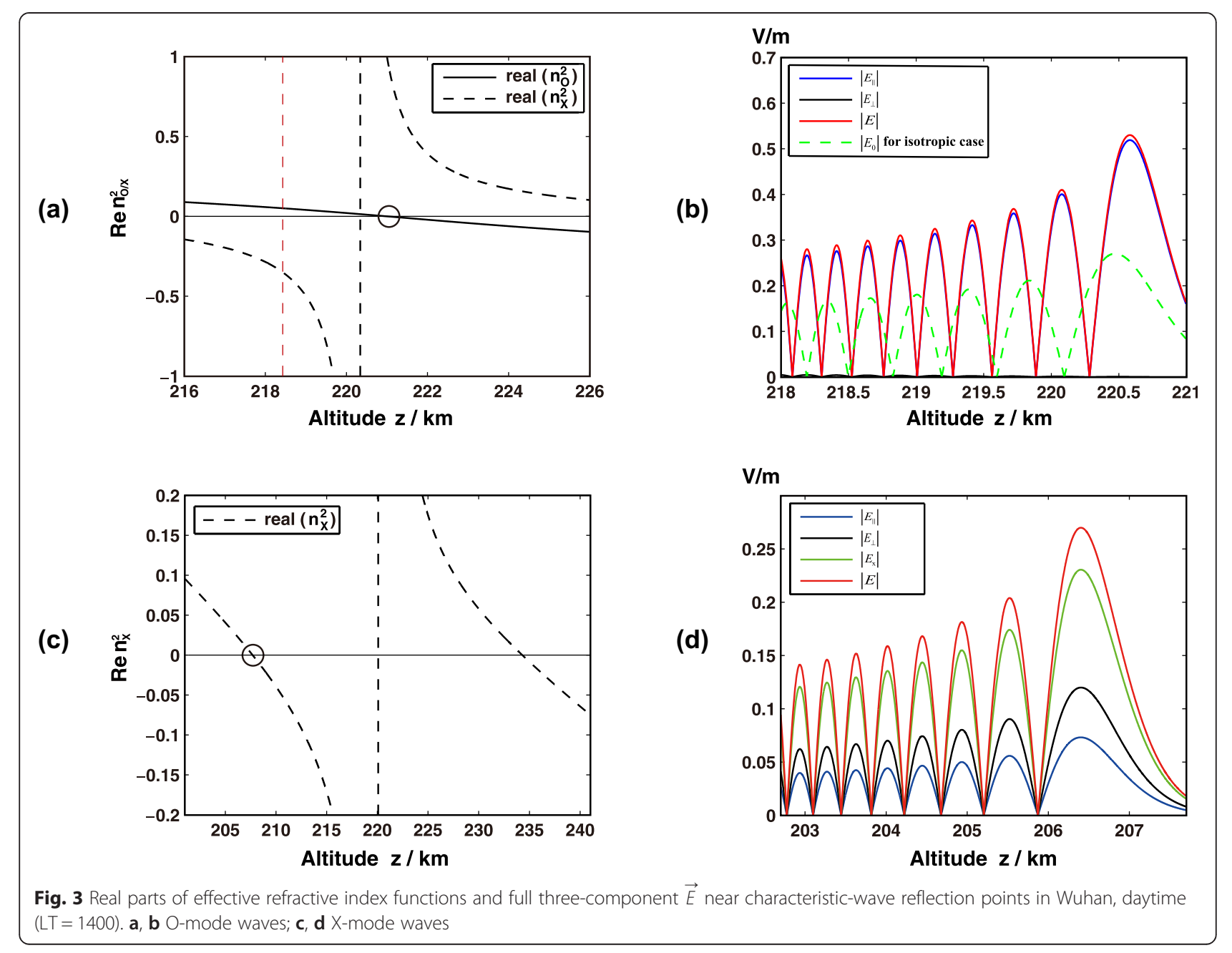

plane $E_{x}$ (the green solid curve in Fig. 2d) is greater than the other two field components in the magnetic meridian plane (the black and blue solid curves in Fig. 2d). In addition, the coefficients obtained in Eq. (34) can be compared to the corresponding coefficients of the O-mode waves in Eq. (31); this may explain why only a reduced field swelling effect is observed for the X-mode waves. As the value of $\theta$ increases as the $Y$ component decreases with decreasing latitude, the swelling effect of the $\vec{E}$ components of the X-mode waves decreases with decreasing latitude.

The red dashed vertical line in Fig. 3a represents the location of the upper-hybrid resonance altitude.

The red dashed vertical line in Fig. 4a represents the location of the upper-hybrid resonance altitude.

The standing wave patterns of the characteristic waves formed near their reflection points in the Wuhan and Haikou areas in daytime $(\mathrm{LT}=1400)$ are illustrated in Figs. 3 and 4, for the parameters given in columns 2 and 4 of Table 1, respectively. Comprehensive analysis considering Figs. 2, 3, and 4 shows that the reflection heights of both characteristic waves increase and the distance between them decreases with decreasing latitude (the black circles in Figs. 3a, c and 4a, c above). In addition, the values of the real parts of $n_{O}^{2}$ and $n_{X}^{2}$ continue to monotonically decrease with height before each reflection point. Furthermore, the distance between the O-mode reflection point $z_{0}$ and the upper-hybrid resonance altitude also decreases with decreasing latitude, as can be concluded from the parameters listed in the last line of Table 1 . The parallel component $E_{\|}$of the $\mathrm{O}$-mode waves continues to play a dominant role in each of the field components and the swelling effect of $\vec{E}$ decreases with decreasing latitude. The amplitude of the $E_{\|}$component in Fig. 4a for the Haikou area is significantly less than that for Alaska, which is shown in Fig. 2a. The swelling factor of the O-mode waves also decreases with decreasing latitude, and the O-mode wave swelling factor in Haikou is only 4.5. Because of the close distance between the upper-hybrid resonance 
(a)

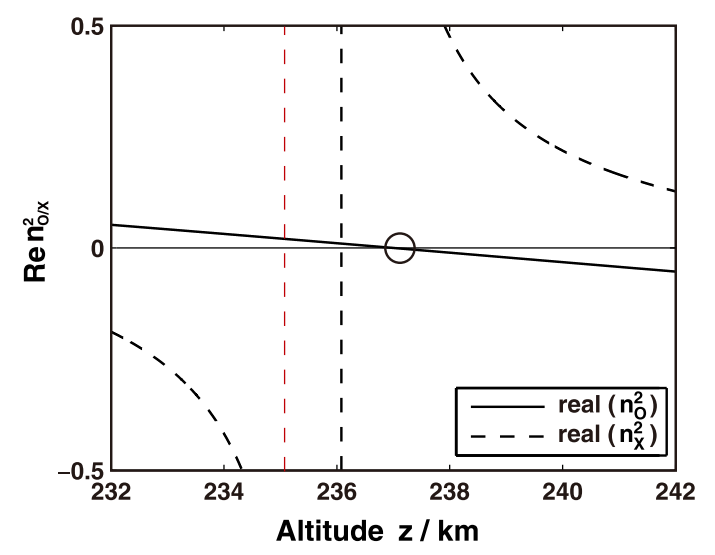

(c)

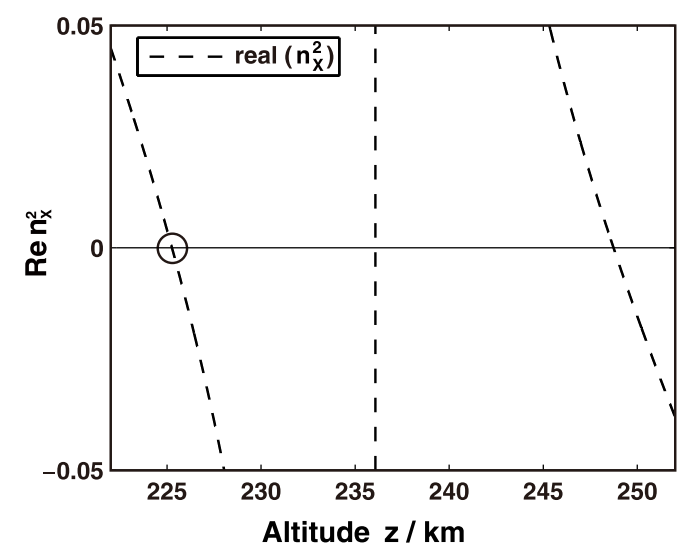

(b)

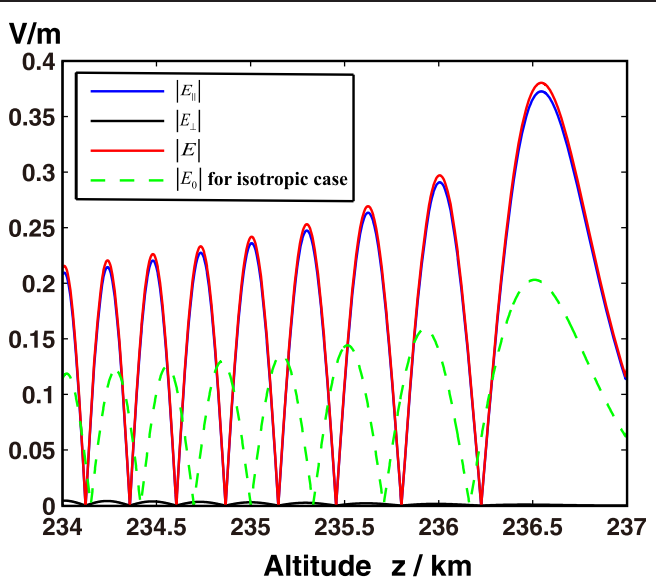

(d)

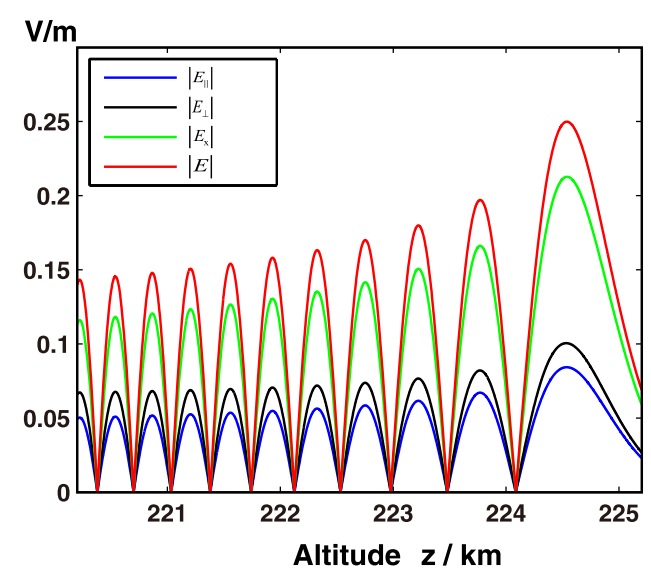

Fig. 4 Real parts of effective refractive index functions and full three-component $\vec{E}$ near characteristic-wave reflection point in Haikou, daytime $(\mathrm{LT}=1400)$. a, b O-mode waves; c, d X-mode waves

altitude and $z_{0}$ in the lower latitude area, the amplitude of $|E|$ at the upper-hybrid resonance altitude is even higher than its value at $z_{0}$. This is because $\vec{E}$ begins to decay before the reflection point is reached.

The electron density and temperature in the background ionosphere, along with the electron collision frequency, may increase with decreasing latitude; this implies that the HF pump-wave emission frequency exhibits a relative growth, while the value of the geomagnetic field may decrease with decreasing latitude. This causes the value of the $Y$ component and the distance between the $\mathrm{X}$ - and $\mathrm{O}$-mode reflection points to gradually decrease with decreasing latitude. The value of $\theta$ may also increase with decreasing latitude. All of the reasons given above may explain why the amplitude of the $E_{\|}$component of the O-mode waves and the swelling effect of $|E|$ gradually decrease with decreasing latitude. Further, the above discussion may also explain why the distance between $z_{0}$ and the upper-hybrid resonance altitude also decreases with decreasing latitude.
For X-mode waves, the amplitude of $E_{x}$ is greater than the other two components in the magnetic meridian plane. In addition, the swelling effect of all components is significantly weaker than in the O-mode wave case. Thus, only a reduced field swelling effect is formed in the vicinity of its reflection point, and the swelling factor for the X-mode waves is less than half that of the $\mathrm{O}$ mode waves.

We have shown the standing wave pattern results for a partially or totally reflected HF wave impinging vertically upon the ionosphere, which were calculated using the "uniform approximation" method within the linear electron density profile at different latitude areas in daytime. However, in addition to the impact of the latitude, the effect of the day-night differences on the ionospheric background parameters is also very obvious. In order to study the impact of these differences on the distribution of the HF pump waves, we present the simulation result for the Wuhan area at night in Fig. 5. The parameters used here are given in column 3 
(a)

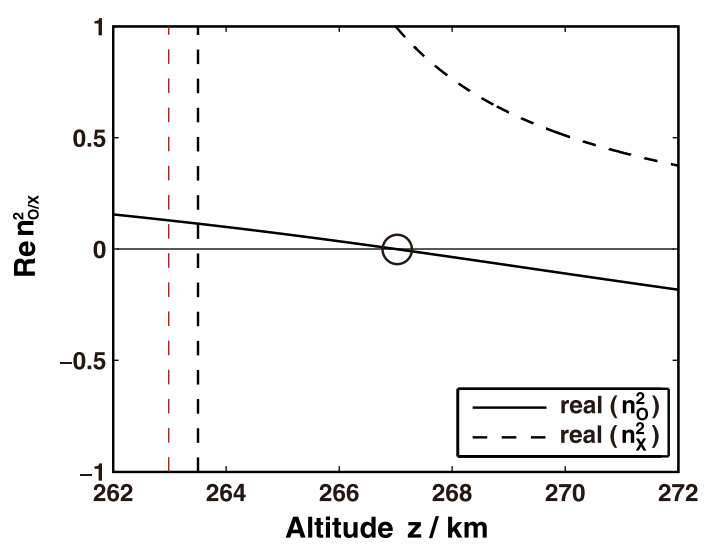

(c)

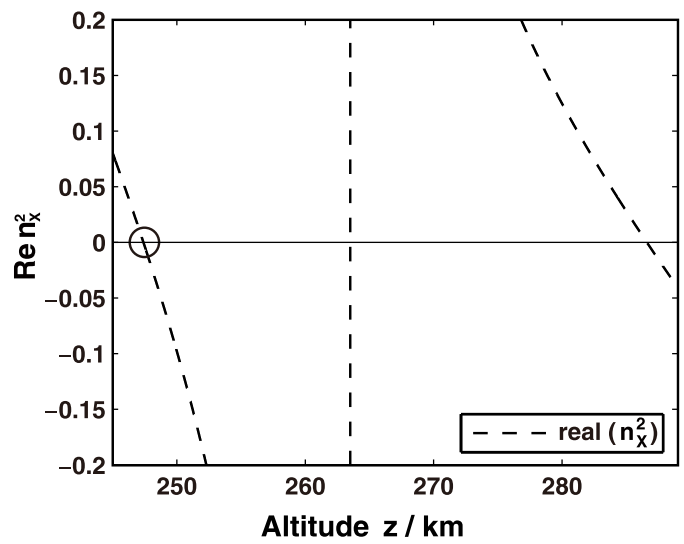

(b)

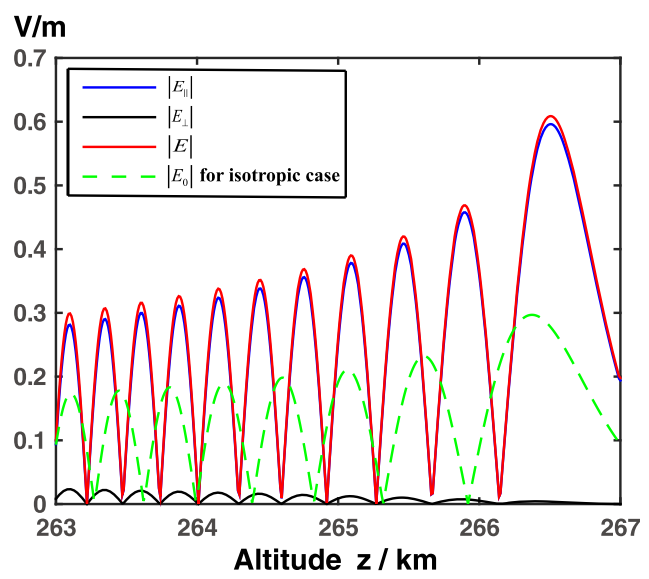

(d)

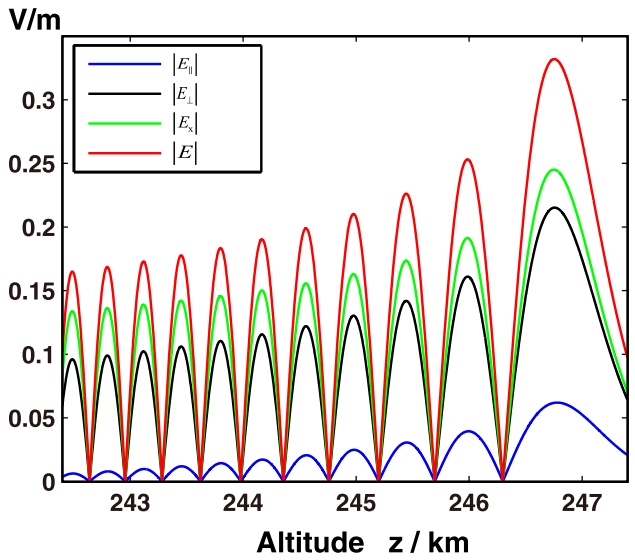

Fig. 5 Real parts of effective refractive index functions and full three-component $\vec{E}$ near characteristic-wave reflection point in Wuhan at night $(L T=0200)$. a, b O-mode waves; c, d X-mode waves

of Table 1. The emission frequency of the pump wave was again set to 0.9 times the local $f_{0} F_{2}$, and the ERP of the pump waves was $10 \mathrm{MW}$.

The red dashed vertical line in Fig. 5a represents the location of the upper-hybrid resonance altitude.

An analysis comparing the results shown in Figs. 3 and 5 shows that the characteristic-wave reflection height at night is significantly greater than the daytime value. In addition, more intensive variations occur in the real part values of $n_{O}^{2}$ and $n_{X}^{2}$ at night. The swelling of the $\vec{E}$ components for the O- and X-mode waves in the vicinity of each reflection points are significantly larger than those during the daytime, and the total $\vec{E}$ intensity can reach significantly higher values at night. As regards the background electron density and electron temperature of the ionosphere, the electron collision frequencies at nighttime are significantly lower than those in daytime; this implies that the HR pumpwave emission frequency has a relatively lower value.
Although the circadian variations of $B_{0}$ are not obvious, the above behaviors cause a sharp increase in the value of the $Y$ component, the distance between the $\mathrm{O}$ and $\mathrm{X}$-mode reflection points, and in the upper-hybrid resonance altitude. In addition, the decrease in the value of the background electron density and the electron temperature of the ionosphere cause a reduction in the background absorption and the energy loss of the HF pump waves. That is, the energy in the vicinity of the reflection points and the field intensity of the standing wave pattern of the characteristic waves are much larger.

\section{Conclusions}

In this paper, we have demonstrated the application of the "uniform approximation" method and the Forsterling equation for investigation of the standing wave pattern of a partially or totally reflected HF wave impinging vertically upon the ionosphere, at different latitudes and also at different local times in a single location. The 
numerical results show that the value of the real part of $n_{O}^{2}$ monotonically decreases with height, while the value of the real part of $n_{X}^{2}$ also monotonically decreases before its reflection point. Further, the distance between the $\mathrm{X}$ - and $\mathrm{O}$-mode wave reflection points decreases with decreasing latitude. It is also found that the swelling of the $\vec{E}$ components of the characteristic waves is much larger and the distance between these maxima is much shorter than in the isotropic case, where the geomagnetic field is neglected. The swelling factors of the pump waves are larger at higher latitudes than at lower ones. Our results suggest that the ionospheric background parameters and the inclination and intensity of the geomagnetic field may have an important effect on the amplitude and spatial distributions of the $\vec{E}$ of HF pump waves.

The ERP value of the HF pump waves is set to $10 \mathrm{MW}$ in our calculation. The $\vec{E}$ at the standing wave maxima near $z_{0}$ reaches a magnitude of hundreds of $\mathrm{mV}$ per meter, exceeding by far the threshold values for certain instabilities (e.g., thermal self-focusing, resonant, and parametric instability) (Kuo, 2015). The intense wave field modifies the plasma density at these maxima. The approximation calculation results in this paper can be used to derive the precise ERP values of HF pump waves used to excite all kinds of instabilities, and also function as a theoretical reference for ionospheric modulation experiments in future.

\section{Competing interests}

The authors declare that they have no competing interests.

\section{Authors' contributions}

$\mathrm{CW}$ performed the derivation of the theoretical equations, wrote the computational program, and drafted the manuscript. CZ conceived the study and proofread the manuscript. ZYZ corrected the errors in the original equations and discussed the results with the authors. XBY helped to solve problems and bugs in the computational program. All authors have read and approved the final manuscript

Received: 21 May 2015 Accepted: 29 June 2015

Published online: 19 August 2015

\section{References}

Abramowitz M, Stegun IA (1972) Handbook of mathematical functions. Dover Press, New York

Banks PM, Kocharts G (1973) Aeronomy, Parts A and B. Academic Press, New York Berry MV, Mount KE (1972) Semiclassical approximations in the wave mechanics. Rep Prog Phys 35:315-397

Budden KG (1966) Radio waves in the ionosphere. Cambridge University Press, New York

Fejer JA (1979) Ionospheric modification and parametric instabilities. Rev Geophys 17:135-153

Fejer JA (1981) Correction to "Ionospheric modification and parametric instabilities". Rev Geophys 19:344-359

Fejer JA, lerkic HM, Woodman RF, Rottger J, Sulzer M, Behnke RA, Veldhuis A (1983) Observations of the HF-enhanced plasma line with a $46.8 \mathrm{MHz}$ radar and reinterpretation of previous observations with the $430 \mathrm{MHz}$ radar. J Geophys Res 88:2083-2092

Field EC, Bloom RM, Kossey PA (1990) lonospheric heating with oblique high-frequency waves. J Geophys Res 95(A12):21179-21186
Ginzburg VL (1970) The propagation of electromagnetic waves in plasmas, 2nd edn. Pregamon, New York

Gondarenko NA, Guzdar PN, Ossakow SL, Brenhardt PA (2003) Linear mode conversion in inhomogeneous magnetized plasmas during ionospheric modification by HF radio waves. J Geophys Res 108(A12):1470-1485. doi:10.1029/2003/A009985

Gondarenko NA, Guzdar PN, Ossakow SL, Brenhardt PA (2004) Perfectly matched layers for radio wave propagation in inhomogeneous magnetized plasmas. J Comput Phys 194:481-504

Gurevich AV (1978) Nonlinear phenomena in the ionosphere. Spring-Verlag, Berlin

Hao SJ, Li QL, Yang JT, Wu ZS (2013) Theory and numerical modeling of under-dense heating with powerful X-mode pump waves. Chinese J Geophys 56(8):2503-2510

Hinkel DE, Shoucri MM, Smith TM, Wagner TM (1992) Modeling of HF propagation and heating in the ionosphere. Rome Laboratory of US Air Force, New York

Hinkel DE, Shoucri MM, Smith TM, Wagner TM, Hansen JD, Morales GJ (1993) Modeling of high-frequency oblique propagation and heating in the ionosphere. Radio Sci 28(5):819-837

Huang WG, Gu SF (2003) The heating of upper ionosphere by powerful high-frequency radio waves. Chin J Space Sci 23(5):343-351

Kuo SP (2015) lonospheric modifications in high frequency heating experiments. Phys Plasma 22:012901. doi:10.1063/1.4905519, 2015

Langer RE (1937) On the connection formulas and solutions of the wave equation. Phys Rev 51:669-676

Lundborg B, Thide B (1985) Standing wave pattern of HF radio waves in the ionospheric reflection region 1. General Formulas Radio Sci 20(4):947-958

Lundborg B, Thide B (1986) Standing wave pattern of HF radio waves in the ionospheric reflection region 2. Applications Radio Sci 21(3):486-500

Miller SC, Good RH (1953) A WKB-type approximation to the Schrodinger equation. Phys Rev 91:174-179

Rietveld MT, Kohl H, Kopka H et al (1993) Introduction to ionospheric heating at Troms $\varphi$ - I. Experimental overview. J Atmos Sol-Terr Phy 55:577-599

Rishbeth H, Garriott OK (1969) Introduction to ionospheric physics. Academic Press, Waltham

Robinson TR (1989) The heating of the high latitude ionosphere by high power radio waves. Elsevier Science Publishers, Amsterdam

Schunk RW, Walker JC (1980) Theoretical ion densities in the lower ionosphere. Space Phys 18:813-820

Stubbe P, Kopka H, Thide B, Derblom H (1984) Stimulated electromagnetic emission: a new technique to study the parametric decay instability in the ionosphere. J Geophys Res 89:7523-7536

Utlaut WF (1970) An ionospheric modification experiments at Troms $\varphi$. J Geophys Res 75(31):6402-6405

Utlaut WF, Cohen R (1971) Modifying the ionosphere with intense radio waves. Science 174:245-254

Utlaut WF, Violette EJ (1972) Further observations of ionospheric modification by a high powered HF transmitter. J Geophys Res 77:6804-6818

\section{Submit your manuscript to a SpringerOpen ${ }^{\circ}$ journal and benefit from:}

- Convenient online submission

Rigorous peer review

- Immediate publication on acceptance

- Open access: articles freely available online

- High visibility within the field

- Retaining the copyright to your article

Submit your next manuscript at $\gg$ springeropen.com 